\title{
La confianza de los estudiantes de traducción en la traducción automática: ¿demasiado buena para ser verdad?
}

\author{
ESTER TORRES-SIMÓN \\ Universitat Rovira i Virgili \\ esther.torres@urv.cat
}

\author{
ANTHONY PYM \\ Universitat Rovira i Virgili \\ anthony.pym@urv.cat
}

\section{Resumen}

Cuando el traductor corrige o "posedita" una traducción automática, no solo trabaja más rápido sino también suele producir una terminología más consistente. Por ello, la mayoría de los programas de formación de traductores incluyen cursos de posedición. Sin embargo, muchos traductores profesionales se oponen al uso de la posedición, lo que da lugar a un conjunto de opiniones negativas sobre la traducción automática que se reproducen en el aula. En este artículo se exponen dos actividades con estudiantes de traducción que incluían posedición y evaluación de traducción automática. Se analizan las actuaciones de los estudiantes en las dos modalidades (traducción totalmente humana frente a la posedición de la traducción automática), para luego compararlas con los comentarios de los propios estudiantes sobre la traducción automática y su experiencia personal tras la interacción. Si bien la mayoría de los participantes reconocieron una mayor eficacia cuando se usa posedición, se posicionaron en contra de la traducción automática, lo que indica un grado significativo de resistencia en el seno de la comunidad de aprendizaje.

\section{Palabras clave}

Traducción automática; aprendizaje de lenguas adicionales; pedagogía; automatización; resistencia a la automatización. 


\title{
Translation students' trust in machine translation: Too good to be true?
}

\begin{abstract}
Advances in neural machine translation have reached the point where professional translators can work faster and produce better terminology when post-editing machine translation as opposed to fully human translation. Most translator-training programs thus include courses in how to post-edit machine translation. Many professional translators, however, are opposed to the use of post-editing rather than fully human translation, resulting in a suite of negative opinions about machine translation within the teaching situation. Here we report on two cases in which classroom activities with translation students involved the post-editing and evaluation of machine translation. We assess the students' actual performances in the two modes (fully human translation as opposed to the postediting of machine translation), which we then compare with the students' comments on machine translation and their personal experience with it. It is found that although the students generally recognized the greater efficiency of post-editing, they nevertheless remained generally opposed to machine translation in principle, indicating extensive resistance within the learning community.
\end{abstract}

\section{Keywords}

Machine translation; additional language learning; classroom activities; resistance to automation.

Recibido el 28/04/2021

Aceptado el 25/05/2021 


\section{Introducción}

Aunque la traducción automática (TA) data de la década de 1950, es en el siglo XXI cuando su calidad ha alcanzado niveles que le permiten rivalizar con la traducción humana. Especialmente desde 2016, con la integración del aprendizaje profundo en los algoritmos estadísticos, la TA neuronal ha dado lugar a aseveraciones de paridad o casi paridad con la traducción humana. Grace et al. (2018) presentan opiniones de expertos en inteligencia artificial que indican que la TA podría sustituir a los traductores profesionales en 2024; la empresa china de traducción automática Baidu afirmó ya en 2016 que su sistema de traducción era "casi tan bueno como el de los seres humanos" (Diño, 2016); Google (2016) calculó que la precisión de su sistema de TA, evaluada por humanos, había mejorado en un $15 \%$ con la introducción del aprendizaje profundo y podía rivalizar con la traducción humana en idiomas como el inglés, el español y el francés; por su parte, Microsoft (Hassan et al., 2018) constató que los crowdworkers bilingües no podían distinguir entre la traducción automática y la humana cuando se les preguntaba por "la semántica del texto de origen".

Sin indagar en el valor científico de dichas afirmaciones (los expertos en inteligencia artificial no conocen necesariamente el uso del lenguaje, y los experimentos con frases aisladas son cuestionados con razón por Läubli, Sennrich y Volk, 2018), aceptamos que la calidad de la TA ha aumentado hasta el punto de que los sistemas gratuitos en línea son utilizados por un enorme número de personas: Google afirmó en 2018 que su sistema procesaba 143 mil millones de palabras al día (Wood, 2018) y Baidu declaró en 2020 que su motor de TA procesaba más de 100 mil millones de bytes al día (Baidu, 2020). Tales cifras implican que los traductores e intérpretes profesionales se ocupan hoy en día de menos del $1 \%$ de todas las traducciones que se realizan en el mundo (Pym y Torres-Simón, 2021).

Vistas estas indudables mejoras y frente al uso generalizado de la TA, cabe esperar que la traducción automática esté cada vez más presente no solo en la formación de traductores, sino en todo tipo de aprendizaje de lenguas adicionales. Como cualquier padre o madre de un estudiante de idiomas debe saber, muchos estudiantes utilizan la TA gratuita fuera del aula, bien como ayuda para los deberes, bien simplemente como diccionario en línea. Sin embargo, ¿qué ocurre dentro de las aulas? ¿Cómo abordan los alumnos las actividades de clase con traducción automática? ¿Se les invita a hacerlo o se les anima a desconfiar de la tecnología? Y lo que es más importante, ¿qué tipo de opiniones tienen los estudiantes ante la afirmación de que la TA rivaliza con la humana? ¿Qué tipo de apoyo o resistencia encontramos ante el uso de esta tecnología?

En este artículo presentamos los resultados de dos actividades de aula llevadas a cabo en una asignatura de traducción que se imparte en un grado de Inglés en Cataluña. Es decir, observamos una situación de enseñanza en la que no se puede evitar la TA y en la que sí se enseña la posedición (la corrección de la TA). Sin embargo, intentamos no orientar a los estudiantes a favor o en contra de la TA; por el contrario, confesamos nuestra relativa ignorancia sobre el verdadero estado de la cuestión, ya que las tecnologías cambian año tras año y muchas de las afirmaciones provienen, obviamente, de partes interesadas. En su lugar, abrimos un espacio donde los estudiantes pueden explorar las tecnologías por sí mismos y extraer sus propias conclusiones. Así pues, abordamos las cuestiones anteriores en dos pasos. En primer lugar, hacemos que los alumnos lleven a cabo un experimento en el que comparan 
su posedición con una traducción publicada y experimentan la traducción y la posedición en textos del mismo tipo y de similar extensión. En segundo lugar, pedimos a los estudiantes que comenten el desarrollo de la actividad, para observar qué tipo de valores y emociones experimentan en su interacción con la traducción automática.

Antes de presentar los resultados creemos importante revisar investigaciones anteriores que pueden ofrecer indicaciones sobre qué se supone que deben encontrar nuestros estudiantes.

\section{2. ¿Puede la traducción automática ayudar a los traductores?}

Hay supuestos fiables de que la posedición (o corrección) de la TA puede ser una forma de trabajo satisfactoria para los traductores tanto profesionales como en formación. Antes de la llegada de la TA neuronal en 2016, diversos estudios ya indicaban que la posedición ofrecía una mayor eficiencia que la versión totalmente manual (por ejemplo, 0’Brien, 2007; Guerberof, 2009; Tatsumi, 2009; Plitt y Masselot, 2010; De Almeida y O’Brien, 2010). Fiederer y O'Brien (2009: 62-63) encontraron que la posedición era ligeramente mejor en cuanto a claridad y precisión, pero peor en términos de estilo. En un estudio realizado en el aula con traducciones del inglés al chino, García $(2010,2011)$ describe casos en que la posedición se valoraba ligeramente mejor que las traducciones totalmente humanas. En su resumen de los estudios anteriores sobre la TA estadística, Koponen (2016) afirmó que la calidad de la TA poseditada puede ser similar a la de la traducción totalmente humana, aunque "la posedición no parece actualmente factible si los poseditores no tienen acceso al texto de origen" (2016: 142). Dado que dichos estudios son anteriores a los avances de la traducción automática neuronal, los beneficios de la posedición deberían ser aún mayores hoy en día.

Dicho lo anterior, hay que tener mucho cuidado con qué se compara en los estudios citados. Lo que está en cuestión no es la calidad de la TA como tal, sino la medida en que el producto de la TA puede ayudar a un traductor humano a producir una traducción que será validada por humanos. Esto significa que los resultados dependen en gran medida de quién lleva a cabo la posedición y de quién hace la evaluación. Si el poseditor es un experto en la materia con años de experiencia de trabajo con TA, las ventajas de la posedición son relativamente fáciles de comprobar: Temizöz (2013), por ejemplo, descubrió que los ingenieros son muy buenos en la posedición de textos de ingeniería. Otro tanto ocurre si el estudio se basa en textos muy técnicos cuya terminología estándar se incluye ya en la base de datos (cf. Plitt y Masselot, 2010).

Por otro lado, si el poseditor no ha recibido una formación especial, o no tiene conocimientos de traducción de alto nivel, o si no cree que la TA debería formar parte de su proceso de trabajo, los resultados pueden no ser tan buenos. El primer informe ALPAC, al principio del desarrollo de la TA, concluyó que "los traductores más rápidos perderán productividad si se les asigna una tarea, mientras que los lentos la ganarán" (1966: 94). García (2010) también comprobó que los traductores menos dotados son los que más se benefician de la posedición. Guerberof (2013) observó que los traductores profesionales tienen sentimientos ambivalentes al poseditar y que su grado de satisfacción depende principalmente de la calidad del resultado de la TA. Teixeira (2014) mostró que los 
traductores perciben las tareas de posedición de forma más positiva cuanto más familiarizados están con el género textual y las tecnologías que se usan, aunque en un experimento con sugerencias de traducción de múltiples fuentes, los traductores profesionales suponían que las traducciones defectuosas procedían de la TA, incluso cuando no era el caso. Por tanto, hay indicios de prejuicios, en parte infundados, en contra de la TA.

Los estudios anteriores aportan, pues, numerosas pruebas de que la posedición puede dar resultados positivos. Sin embargo, también indican que las creencias de los traductores sobre la TA pueden ser un factor inhibidor.

LeBlanc $(2013,2014)$ estudió el uso de las tecnologías de la traducción en el lugar de trabajo y reveló que la insatisfacción de los traductores no se debía tanto a las herramientas en sí mismas como a las prácticas empresariales que se han construido con base a lo que supuestamente aportan. La posibilidad de medir la producción de forma mecánica hace que el traductor tenga que cumplir con los requisitos de productividad: la calidad se redefine como número de palabras por unidad de tiempo. Además, la frecuente obligación de reciclar traducciones anteriores se percibe negativamente como una alteración de las prácticas laborales anteriores. Otros estudios constatan que los traductores se preocupan por la caída de las tarifas salariales asociadas a la posedición (Vieira, 2018): los beneficios financieros de las tecnologías tienden a ir a las grandes empresas y no a los traductores (Pym y TorresSimón, 2021). Dicha insatisfacción también se asocia a cierta preocupación por la reducción de la interacción humana en el lugar de trabajo (Cadwell et al., 2016; Cadwell, O’Brien y Teixeira, 2018) y a la pérdida de la voz humana en los textos (una inquietud que se remonta a Bédard, 2000), especialmente en las traducciones literarias (Kenny y Winters, 2020).

Considerando estas situaciones, la reticencia detectada en estudios como los de Guerberof (2013) y Teixeira (2014) podría ser una manifestación más de una generación consolidada laboralmente que se aferra a las tecnologías, a los flujos de trabajo y a las relaciones interpersonales que las asentaron en dicha situación de poder. Por tanto, la resistencia a la TA tendría su base en una generación no solo de traductores de éxito, sino también de profesores de traducción con experiencia. Frente a esos traductores consolidados y en interacción con esos profesores experimentados, hay una nueva generación de principiantes. ¿Qué opinan estos últimos de las tecnologías de la traducción?

Los datos parecen contradictorios en este punto. En un estudio en que se invitó a traductores y a estudiantes de traducción a escribir cartas de amor o de odio a diversos programas de memoria de traducción, Koskinen y Ruokonen (2017) descubrieron que los estudiantes de traducción mostraban mayor ansiedad por los efectos de la tecnología que los traductores veteranos. Por otro lado, Dillon y Fraser (2006) y Katan (2011) observaron que los traductores más jóvenes estaban más abiertos a las nuevas tecnologías de la traducción que los traductores de más edad. También hay que tener en cuenta cierta dimensión geoeconómica: en un estudio comparativo de las preguntas que se plantean los estudiantes sobre la traducción, Pym y Torres-Simón (2016) descubrieron que los traductores en formación de Monterey (California) veían las tecnologías de la traducción de forma mucho más positiva que los alumnos correspondientes de Viena, presumiblemente porque los primeros estudiaban cerca de fuentes de empleo futuras como Silicon Valley.

En resumen, cabe esperar que las nuevas tecnologías de la traducción resulten atractivas para quienes más pueden beneficiarse de ellas (los no traductores, los que tienen menos 
conocimientos lingüísticos o los traductores en prácticas que buscan empleo en empresas tecnológicas) $\mathrm{y}$, en cambio, sean menos atractivas para quienes se han consolidado con tecnologías anteriores (los traductores profesionales de éxito, sobre todo los que trabajan con textos literarios; quizá también los profesores con experiencia). Entre unos y otros, la posición de los traductores noveles no parece estar definida. Por un lado, es de suponer que han crecido con las nuevas tecnologías y están relativamente acostumbrados a los avances tecnológicos. Por otro, tienen poco capital cultural que defender y, por tanto, pueden sentirse más amenazados por las novedades.

¿Cómo negocian los estudiantes estas cuestiones? Algunos estudios han intentado responder esta pregunta.

\section{La posedición en la clase de traducción}

Los programas de formación de traductores han incorporado progresivamente las tecnologías de la traducción al menos desde la década de 1990 (Hartley y Schubert, 1998). En 2018 se informó de que más del 90 \% de los programas de la European Masters of Translation (red de másteres europeos de traducción) enseñaban nuevas tecnologías (Rothwell y Svoboda, 2018). La TA también se ha utilizado en el aprendizaje de lenguas adicionales en décadas similares (cf. Lewis, 1997; Niño, 2008, 2009; Ducar y Schocket, 2018), aunque con una historia más accidentada: la resistencia basada en las pedagogías de inmersión ha sido contraria a cualquier uso de la traducción, no solo a la TA (véase, por ejemplo, Haugh 2017, quien afirma erróneamente que los sistemas de TA no son conscientes de que las diferentes lenguas recortan el espacio semántico de diferentes maneras). Con todo, hoy en día se reconoce que los estudiantes utilizan la TA, de modo que no solo deben aprender a utilizarla bien (véase, por ejemplo, Clifford, Merschel y Munné, 2013; Jolley y Maimone, 2015), sino que todos los actores implicados en la acción docente deben adaptarse a las nuevas relaciones de poder que surgen del uso de estas tecnologías - el profesor ya no es el único actor que posee el conocimiento de los idiomas (Case, 2015)—.

En este estudio, sin embargo, nos centramos específicamente en las clases de traducción, donde los argumentos a favor de la enseñanza de las tecnologías de la traducción son obviamente mucho más contundentes y donde la enseñanza transmisionista hace tiempo que se ha cuestionado (Kiraly, 2000). Las principales tecnologías que se enseñan a los traductores en formación son las memorias de traducción (Trados, Memsource, MemoQ y similares), la integración de la TA y, en algunos casos, los sistemas de gestión de terminología y de contenidos. Es posible y pedagógicamente sensato enseñar estas tecnologías por separado: Trados y MemoQ, por ejemplo, pueden enseñarse sin ningún complemento de TA. Sin embargo, los programas gratuitos de memoria de traducción en línea incorporan actualmente fuentes de TA, de manera que, aunque pueda deshabilitarse, los estudiantes tienden a experimentar ambas cosas juntas y les resulta difícil separarlas conceptualmente.

Los pocos estudios sobre el uso de la TA en clases de traducción se ven obstaculizados por las normas del género: prácticamente todos reportan historias de éxito, ya que ningún profesor quiere confesar sus fracasos. Lo mismo ocurre en el campo de la tecnología. Las descripciones de lo que se hace en las distintas asignaturas de tecnología de la traducción 
(por ejemplo, O'Brien, 2002; Kenny y Doherty, 2014; Guerberof y Moorkens, 2019) muestran los valores positivos de las sinergias entre el ser humano y el ordenador, la exploración dinámica, la interacción grupal y el empoderamiento de los alumnos, extrañamente con escasa mención a algún tipo de resistencia por parte de los estudiantes.

Otros estudios, sin embargo, sí que atienden a la perspectiva de los estudiantes. Gaspari (2001) impartió una asignatura de TA a un grupo de 38 traductores en formación. Al principio del curso, el $10 \%$ de los estudiantes veía la TA como una amenaza a los traductores humanos, mientras que el 27 \% no tenía opinión. Al final de la asignatura, solo un estudiante pensaba que la TA era una amenaza, mientras que dos (5\%) seleccionaron "no sabe". Media generación más tarde, Moorkens (2018) informa de manera similar sobre una asignatura de traducción asistida por ordenador. A los 48 estudiantes de grado se les pidió específicamente que comparasen los resultados de la TA estadística con los de la TA neuronal. Alrededor del $93 \%$ de los estudiantes expresaron su preferencia por la TA neuronal, aunque todos los estudiantes parecían haber tomado conciencia de que sigue habiendo traducciones erróneas y (especialmente) omisiones en la neuronal. Moorkens no incluyó ninguna pregunta sobre la visión de la tecnología en general - su atención se centró en el rendimiento y la detección de errores-, pero generaliza que los estudiantes "disfrutaron de la posedición de la TA, y lo encontraron más fácil que traducir desde cero" (2018: 9).

En consonancia con el género, estos dos informes son muy optimistas sobre la tecnología. Conocer la tecnología es amarla, o así parece. Ahora bien, ¿realmente los estudiantes de traducción no muestran ningún tipo de resistencia ante estas tecnologías?

\section{Propuesta educativa}

Para intentar resolver esta cuestión, analizamos a continuación las respuestas a dos tareas realizadas de modo virtual en el año académico 2020-2021 en la asignatura optativa de Traducción Directa I del grado de Inglés de la Universitat Rovira i Virgili. Esta asignatura de traducción del inglés al español o catalán es al menos la segunda que realizaron sus participantes. Todos los participantes tenían el español o el catalán como lengua A y el inglés como lengua $\mathrm{B}$.

La propuesta educativa está diseñada en dos partes. La primera parte (4.1) invita a los participantes a teorizar sobre la TA, con el objetivo de que sopesen sus ventajas y limitaciones. La segunda parte (4.2) ahonda de forma deliberada en una de las principales ventajas de la TA, el aumento de la eficiencia, para luego recabar la opinión del participante sobre su uso, con el objetivo de mostrar posibles contradicciones y prejuicios. El enunciado de las tareas se reproduce en el Anexo 1 y el Anexo 2.

Las respuestas se compartieron de manera individual en formato Word, con excepción de la tercera parte de la tarea 4.1, que se compartió en el foro de Moodle. Este foro estaba programado para que el participante no viera las respuestas de los compañeros hasta realizar su propia intervención.

Dado el tamaño reducido de la muestra (28 participantes), el análisis cualitativo de las respuestas se hace de manera inductiva, codificando manualmente las respuestas para luego 
agrupar las principales ideas. La tarea y los resultados de las respuestas se analizan a continuación.

\subsection{Tarea de adivinar el proceso}

En la tarea 4.1 se pidió a los participantes que completaran una tarea de TA y posedición para luego comparar su propuesta con la traducción publicada. Para seleccionar los textos, los participantes tenían que visitar la web de la revista en línea Tradumàtica, donde se publican resúmenes en inglés, catalán y español. Cada participante realizó los pasos siguientes:

- Seleccionar uno o más resúmenes en inglés, de modo que sumaran al menos 400 palabras, que constituyen el texto de partida.

- Realizar la traducción automática y posedición del texto de partida a la lengua A del participante (catalán o español).

- Comparar la traducción resultante con el resumen en español o catalán que se ha publicado en la misma revista.

- Decidir si el resumen publicado parece ser traducción humana o más bien el resultado de la TA.

Es importante subrayar que el resumen que se ha publicado en la revista podría ser el resultado de la traducción humana, de la TA, de la TA más posedición o de un texto original. Lo que nos interesa no es cómo se ha producido el texto (de hecho, no lo sabemos), sino qué opina el participante sobre su posible procedencia.

Tras realizar los pasos mencionados, el participante tenía que contestar a cuatro preguntas:

1. ¿Merece la pena utilizar la traducción automática? ¿O habría sido mejor traducir sin traducción automática?

2. ¿Piensas que la traducción publicada se hizo con traducción automática? Si es así, ¿cómo lo sabes?

3. Enumera al menos cuatro tipos de correcciones que has hecho con la posedición y que no harías normalmente al revisar un texto.

4. Propón una teoría sobre por qué la traducción automática necesita estos tipos de correcciones.

La tarea fue completada por los 28 participantes.

\subsection{Resultados de la posedición de resúmenes}

En respuesta a la primera pregunta, la mayoría de los estudiantes (61\%) consideraron que sí que valió la pena poseditar la TA (Tabla 1). 
Tabla 1. ¿Mereció la pena usar la traducción automática? $(n=28)$

\begin{tabular}{lcc}
\hline Respuesta & Valor absoluto & Porcentaje \\
\hline Sí & 17 & 61 \\
No & 7 & 25 \\
Depende & 2 & 7 \\
No contesta & 2 & 7 \\
\hline
\end{tabular}

A partir de los resúmenes analizados, la mayoría consideró que la traducción publicada era una traducción humana (18 participantes, $64 \%$ ) y el resto, que era TA (10 casos, $36 \%$ ). Al intentar justificar si se había utilizado la TA, los participantes ofrecieron una serie de teorizaciones sobre esta última, de las que se deducen los indicadores recogidos en la tabla 2.

Tabla 2: Indicadores de diferenciación de TA

\begin{tabular}{lc}
\hline Indicadores & Iteraciones \\
\hline Errores de traducción & 11 \\
Estructura gramatical & 4 \\
Expresiones (léxico) & 2 \\
Calidad de la traducción & 2 \\
No se ofrecen razones & 4 \\
Parafraseo & 2 \\
Referencias culturales & 1 \\
Longitud de la frase & 1 \\
No sabe & 1 \\
\hline
\end{tabular}

En general, se asocia la TA a mala calidad o a errores de traducción. Por ejemplo, el participante número 12 (P12) opina:

P12: I do not think the published translation was done with MT because some of the expressions, words, word structure, among others, would have been translated inaccurately. ${ }^{1}$

O sea, si la traducción es buena, no puede ser automática. Por su parte, la traducción humana se asocia a la originalidad, la adición de palabras y a una mayor desviación con respecto al texto de partida. Es decir, se presume que la TA es literal:

\footnotetext{
${ }^{1}$ No creo que la traducción publicada se hiciera con TA porque algunas de las expresiones, palabras y estructura de las palabras, entre otros, no se habrían traducido adecuadamente. (Aquí y en adelante, la traducción es nuestra.)
} 
P6: I believe the translation published was not done with MT because of the order of the words and the expressions used. Also, there are expressions that are not in the original text. ${ }^{2}$

P4: The translator uses words that MT systems do not have in their system. ${ }^{3}$

P24: I feel that the translated texts are way too different from the English ones in terms of the structures of some sentences, and in the translation of the acronyms. ${ }^{4}$

En cierta manera, las anteriores opiniones encierran la crítica de que no ha habido una posedición extensa tras la TA.

Al reflexionar sobre el tipo de correcciones que se han hecho en la posedición preliminar, se mencionan desde errores que raramente cometería un traductor humano (errores graves de traducción, repetición del mismo término en una secuencia) o errores básicos que se solucionarían en revisión (uso de mayúsculas, puntuación, uso incorrecto de pronombres) hasta más avanzados, como errores en la traducción de frases hechas o ajustes de registro y estilo (ver listado completo en la Tabla 3).

Esta clasificación, sin embargo, no se presenta como un análisis exhaustivo de la calidad de una TA, sino más bien una consideración de qué tipo de errores los estudiantes no cometerían. Es decir, al pedir una selección limitada de errores, se tiende a resaltar los más habituales.

Tabla 3. Clasificación de errores hallados

\begin{tabular}{lc}
\hline \multicolumn{1}{c}{ Tipo de error } & Iteraciones \\
Errores léxicos & 18 \\
Uso del artículo & 17 \\
Colocaciones & 11 \\
Género nominal & 10 \\
Orden de la frase & 8 \\
Uso de la mayúscula & 7 \\
Acrónimos & 6 \\
Uso verbal (incluye uso de pasiva) & 6 \\
Puntuación & 5 \\
Referencias culturales o idiomáticas & 4 \\
Repetición innecesaria & 3 \\
Registro y estilo & 3 \\
Posesivos & 1 \\
Pronombres & 1 \\
Mezcla de variedades del español & 1 \\
\hline
\end{tabular}

\footnotetext{
${ }^{2}$ Creo que la traducción publicada no se hizo con TA por el orden de las palabras y las expresiones utilizadas. Además, hay expresiones que no están en el texto original.

${ }^{3}$ El traductor usa palabras que los sistemas de TA no tienen en su sistema.

${ }^{4}$ Me da la sensación de que los textos traducidos son demasiado diferentes de los textos ingleses en términos de estructuras de la misma frase y en la traducción de acrónimos.
} 
Como respuesta a la cuestión final sobre por qué la TA da lugar a ciertos tipos de errores, en algunos casos se resume simplemente la respuesta a la pregunta anterior. En otros casos, la reflexión se transforma en teorización.

Tabla 4. Resumen de justificación de errores de la TA

\begin{tabular}{lc}
\hline Conceptos asociados con TA & Iteraciones \\
\hline Problemas relacionados con la pragmática & 19 \\
TA traduce las palabras literalmente & 12 \\
TA no está a la altura de un traductor humano & 11 \\
Errores de género & 5 \\
TA necesita actualizarse con regularidad & 3 \\
Problemas de puntuación & 2 \\
La calidad de la TA es baja & 2 \\
\hline
\end{tabular}

En general, se centra en la incapacidad de elegir opciones léxicas adaptadas al contexto (19) o de superar la literalidad (12):

P03: There are some expressions that only humans can understand. [...] Sometimes one word is more adequate than another and machines are not always able to choose the correct one. ${ }^{5}$

P28: Many times if a word has various meanings, MT has more issues with which one is adequate for the context. ${ }^{6}$

Es decir, la literalidad presente en las traducciones muestra una antropomorfizada falta de decisión:

P13: It is still quite limited when it comes to breaking fixed structures in order to make the text more readable and comprehensible for the reader. [...] Moreover, I also think that MT gets some other things wrong because it hasn't got its own criteria or the capacity to make translation decisions. ${ }^{7}$

Por otra parte, se entiende que la TA no conlleva una renovación de la lengua. Dada su evolución, es necesario actualizar las bases de datos:

\footnotetext{
${ }^{5}$ Hay algunas expresiones que solo pueden entender los humanos (...). Algunas veces una palabra es más adecuada que otra y las máquinas no son siempre son capaces de elegir la correcta.

${ }^{6}$ Muchas veces, si una palabra tiene más de un significado, la TA tiene más problemas para elegir cuál es el adecuado al contexto.

${ }^{7}$ Aún es muy limitada cuando tiene que dividir estructuras fijadas para que el texto sea más legible y comprensible para el lector. Además, también creo que la TA hace mal otras cosas porque no tiene su propio criterio o la capacidad de tomar decisiones de traducción.
} 
P04: Language changes all the time, words get new meanings. ${ }^{8}$

Por todo ello, se considera que la propuesta que surge de la TA es de mala calidad, de modo que la TA queda lejos de sustituir a los traductores:

P02: But no machine translation software will ever be able to replace translators. ${ }^{9}$

P10: It is impossible to configure all the problems that language has into the machine. ${ }^{10}$

No deja de llamar la atención el que se haga hincapié en que los traductores no podrán ser reemplazados por un traductor automático, que es una idea que nunca se ha transmitido en nuestra aula de traducción.

\subsection{Tarea de comparar procesos}

Si la tarea 4.1 se concebía como una introducción a la TA y proponía una reflexión desde el punto de vista de las diferencias entre uno y otro tipo de traducción y los errores distintivos - y fácilmente localizables - de la TA, la tarea 4.2 fuerza a los estudiantes a tener en cuenta el factor tiempo.

La tarea se basa en un texto de carácter formal: la introducción a una normativa de la Unión Europea, en inglés. El texto se organiza en párrafos, de los cuales cada participante debía traducir una selección 1) manualmente, 2) con TA y posedición y 3) con herramientas de traducción con memoria de traducción (Matecat). Se pide que se cronometren los tres métodos de trabajo y se evalúe su dificultad. Finalmente, se pide una reflexión sobre el uso de herramientas y de la tecnología en la traducción.

This task has three parts. Submit one file answering all three parts. Then provide a conclusion on the importance of technologies in translation.

Part 1: Open the text European Regulations and translate the first article. How long did it take? Was it easy or difficult?

Part 2: Now, pass the second article through Google Translate. Then post-edit it. How long did it take? Was it easy or difficult?

Part 3: Use Matecat to translate the remaining articles, using the attached TMX file. How long did it take? Was it easy or difficult?

La tarea se plantea de esta manera para intentar aclarar posibles confusiones entre traducción automática (TA) y memorias de traducción (TAO). La formación en Matecat había mostrado cómo visualizar la procedencia de las propuestas ofrecidas por el sistema. Sin embargo, en la práctica se muestra que la distinción no es clara. Por ello, presentamos las respuestas relativas a la parte 1 y 2 .

\footnotetext{
${ }^{8}$ La lengua cambia todo el tiempo, las palabras obtienen nuevos significados.

9 Pero ningún software de TA podrá jamás reemplazar a los traductores.

10 Es imposible configurar en la máquina todos los problemas que presenta la lengua.
} 
La tarea fue completada por 26 estudiantes, pero solo 23 proporcionaron datos exactos sobre tiempo y dificultad.

\subsection{Resultados de la comparación de procesos}

El fragmento 1 constaba de 61 palabras, mientras que el fragmento 2 tenía 89. Cuando los tiempos se ajustan por el número de palabras, la posedición (tarea 2) sería netamente más rápida que la traducción sin TA (tarea 1) a juzgar por las medias (14,61 minutos frente a 21,30 ). Hay, sin embargo, una variación considerable en relación con la eficacia con que los participantes utilizan la tecnología (Figura 1), lo que hace que dicha diferencia no resulte significativa $(\mathrm{p}=0.171)$.

Figura 1. Tiempo invertido en cada tarea $(n=23)$

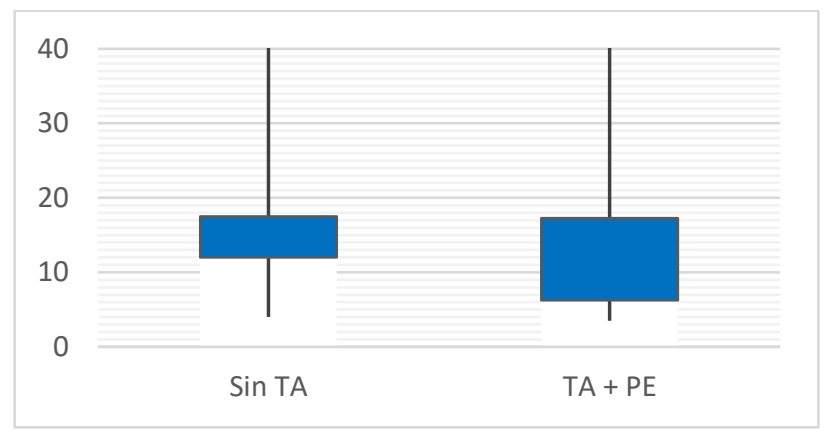

Respecto a la dificultad, en la valoración de las respuestas se asigna un nivel de dificultad de 1 a 5 , en que 1 es muy fácil y 5 es muy difícil. Si se atiende a los resultados (Tabla 5), la posedición de la TA parece haber sido algo más fácil que la traducción humana.

Tabla 5. Dificultad por tarea $(n=26)$

\begin{tabular}{ccc}
\hline Dificultad & Sin TA & TA y posedición \\
\hline 1 & 5 & 6 \\
2 & 11 & 8 \\
3 & 7 & 11 \\
4 & 3 & 1 \\
5 & 0 & 0 \\
\hline Media & 2.31 & 2.27 \\
\hline
\end{tabular}

Las diferencias de tiempo en la realización de una misma tarea van muy ligadas a cuán concienzudo es el participante: en la tarea sin TA, hay estudiantes que reportan no haber usado apenas el diccionario y otros que crearon un glosario antes de iniciar la traducción de las apenas 100 palabras. 
Lo que resulta más significativo son las explicaciones de los participantes que tardaron más tiempo en la posedición de la TA que en la traducción sin TA. Se pueden leer opiniones como las siguientes:

P21: At first one may think that using Google Translate saves time, but in my case, it made me doubt a myriad of times. ${ }^{11}$

P16: The fact that I do not see errors has made me distrustful, and therefore I have had to read the text many times to try to see errors. ${ }^{12}$

Es decir, tardaron más tiempo en completar la tarea porque no se podían creer que el resultado hubiera sido de tanta calidad.

Por otra parte, no es sorprendente que la traducción sin apoyo tecnológico pueda ser la más difícil: al tratarse de un texto formal (semilegal), es posible que los participantes no estuvieran familiarizados con la terminología ni con la estructura textual. De hecho, los comentarios ofrecidos libremente por los estudiantes refuerzan el rol de la terminología en la dificultad del texto. Por ejemplo, un participante indica sobre la traducción humana:

P13: I also think that if the text had been longer (and consequently, had had more technical terminology) it would have been harder for me, and would also have taken me more average time to translate it. ${ }^{13}$

Es la reflexión, una vez más, lo que permite ver el posicionamiento de los participantes. Aproximadamente el $62 \%$ aprecian las ventajas del uso de las tecnologías. Sin embargo, casi el $80 \%$ le ven desventajas (véase Tabla 6). Como ya indica la cifra de estos porcentajes, estas ideas se combinan a veces, en un intento de explicar una realidad.

Tabla 6 . Ideas básicas en las conclusiones $(n=26)$

\begin{tabular}{lc}
\hline Conclusiones (ideas básicas) & Incidencias \\
\hline La TA acelera y facilita el trabajo & 15 \\
La TA no es 100\% de confianza & 11 \\
La TA jamás sustituirá a los humanos & 9 \\
MateCat es mejor que solo TA & 3 \\
La TA mejora la calidad de la traducción & 2 \\
Es importante tener un plan de documentación & 1 \\
La TA mejora el precio de una traducción & 1 \\
La TA no es buena & 1
\end{tabular}

${ }^{11} \mathrm{Al}$ principio se podría creer que usar Google Translate ahorra tiempo, pero, en mi caso, me hizo dudar multitud de veces.

${ }^{12}$ El hecho de no ver errores me hizo desconfiar, y por tanto he tenido que volver a leer el texto muchas veces para intentar ver errores.

13 También creo que si el texto hubiera sido más largo (y por tanto hubiera tenido más terminología técnica) habría sido más duro para mí y me hubiera tomado más tiempo de media traducirlo. 
De hecho, solo un $25 \%$ de los participantes (7) valora positivamente la TA sin añadir ninguna muletilla aclaratoria. Es decir, los participantes que consideraron la tarea 1 más difícil que la 2 consideran que la TA facilita y agiliza el trabajo, pero añaden la muletilla de que no es fiable o de que no reemplazará a los humanos. Por ejemplo:

P6: I think technologies are a positive thing that can help us and they will be even better because they are improving every day. Although behind a good translation, there will have to be always a person. ${ }^{14}$

Otro participante, en la misma situación, es más claro:

P2: MT will never achieve it without translators' cooperation. ${ }^{15}$

Curiosamente, esta resistencia a aceptar la TA es clara incluso cuando todo el proceso y el resultado se valoran de forma positiva:

P5: [MT] was not as bad as I imagined. [...] I didn't really find any relevant mistake when post-editing it. Even though [sic], we always have to check the result first since it is not a hundred percent reliable. ${ }^{16}$

Esta visión previa llega a tal punto que impide que el resultado se considere positivamente:

P10: Creo que ha sido más difícil que la parte anterior porque ha supuesto más trabajo, ya que Google Traductor no siempre traduce los textos de manera correcta y se tiene que leer, editar, buscar información para traducir correctamente y después cambiarlo.

En resumen, no hay un posicionamiento claro en contra de la tecnología, ni específicamente en contra de la TA, pero tampoco claramente a favor. En general, la reticencia a aceptar que un uso adecuado de las nuevas tecnologías es una mejora para la profesión sigue patente en las respuestas, y esto tiene consecuencias en cómo se usan. Una opinión excesivamente negativa sobre la TA fuerza a invertir mucho tiempo en posedición, lo que va contra una de las principales ventajas del uso de TA: la velocidad de traducción. En el caso analizado, además, llama la atención que los profesores de traducción que han tenido los estudiantes apoyan el uso de todo tipo de tecnologías, incluida la TA, de modo que tal posicionamiento negativo se puede considerar externo al aula de traducción.

\footnotetext{
${ }^{14}$ Creo que las tecnologías son algo positivo que pueden ayudarnos y que serán aún mejores porque mejoran cada día. Aunque, tras una buena traducción, siempre habrá una persona.

${ }^{15}$ La TA nunca lo conseguirá sin cooperación humana.

${ }^{16}$ [La TA] no fue tan mala como la imaginé. [...] Ni siquiera encontré ningún error relevante al poseditar. Aun así siempre tenemos que comprobar los resultados primero puesto que no es $100 \%$ fiable.
} 


\section{Conclusión}

En línea con estudios anteriores, parece ser que, efectivamente, conocer la tecnología es llegar a amarla. Sin embargo, siguiendo con la analogía, no es tanto un amor a primera vista como una cita a ciegas. Los estudiantes se aproximan a la traducción con una opinión formada previa, que en general es escéptica.

Por ello es importante permitir que sean los estudiantes los que conozcan de primera mano las ventajas e inconvenientes de la TA, eso sí, tras una formación específica que les ayude a saber valorar qué es una traducción de calidad.

Sería interesante replicar la tarea cambiando el perfil del estudiante, puesto que las opiniones y expectativas del alumnado de un grado de inglés podrían ser diferentes de la visión de estudiantes de traducción, ya sean de grado o de máster, que podrían tener mayor conciencia de la situación de la profesión y sus tecnologías.

En cualquier caso, como formadores que trabajan en este ámbito, somos muy conscientes de que las tecnologías están en constante evolución. Por tanto, no pretendemos tener conocimientos fijos sobre su valor o eficacia: en clase no decimos si la TA es buena o mala; es algo que cada alumno debe descubrir por sí mismo. Nuestra tarea pedagógica es poner en marcha el proceso de descubrimiento. Dicho esto, parece que todavía existe una considerable resistencia a las nuevas tecnologías, hasta tal punto que el paso más importante, desde el punto de vista pedagógico, podría ser que el estudiante se desprenda de los prejuicios previos.

\section{Anexo 1. Tarea 4.1. (Traducción Directa I, optativa, grado de Inglés)}

\subsection{MT and postediting}

1. Go to https://revistes.uab.cat/tradumatica and select abstracts in English so that you have at least 400 words. (You may have to look at other issues of the journal to find suitable abstracts.)

2. Run them through an MT system to convert them into your A language.

3. Now postedit the 400 words, using track changes. Keep a track of how long you spend doing this.

4. Compare your postediting with the version of the abstract in your A language that is published in the journal.

Prepare brief answers to the following questions, and submit them here:

1. Was it worth using MT, or would it have been better to translate from scratch?

2. Do you think the published translation was done with MT? If so, how can you tell?

3. List at least four kinds of corrections that you make with postediting that you would not normally make when revising a text, and give two examples of each. (Please post these to the Forum Thread on Moodle.)

4. Propose a theory on why MT gets these things wrong. (To check this, you might want to try a few back-translations in your MT.) 


\section{Anexo 2: Tarea 4.2. (Traducción Directa I, optativa, Grado de Inglés).}

\section{Task 4.2. Translation technologies}

This task has three parts. Submit one file answering all three parts. Then provide a conclusion on the importance of technologies in translation.

Part 1: Open the text European Regulations and translate the first article.

How long did it take? Was it easy or difficult?

Part 2: Now, pass the second article through Google Translate. Then post-edit it.

How long did it take? Was it easy or difficult?

Part 3: Use Matecat to translate the remaining articles, using the attached TMX file.

How long did it take? Was it easy or difficult?

\section{Referencias bibliográficas}

BAIDU (2020). Baidu Announces Third Quarter 2020 Results. https://ir.baidu.com/newsreleases/news-release-details/baidu-announces-third-quarter-2020-results

BÉDARD, C. (2000). Mémoire de traduction cherche traducteur de phrases. Traduire, 186, 4149.

CADWELL, P., CASTILHO, S., O’BRIEN, S., \& MITCHELL, L. (2016). Human factors in machine translation and post-editing among institutional translators. Translation Spaces: A Multidisciplinary, Multimedia, and Multilingual Journal of Translation, 5(2), 222-243.

CADWELL, P., O'BRIEN, S., \& TEIXEIRA, C. S. C. (2018). Resistance and accommodation: factors for the (non-) adoption of machine translation among professional translators. Perspectives, 26(3), 301-321.

CASE, M. (2015). Machine translation and the disruption of foreign language learning activities. eLearning Papers, 45, 4-16.

CLIFFORD, J., MERSCHEL, J, \& MUNNÉ, J. (2013). Surveying the landscape: What is the role of machine translation in language learning? @tic. Revista d'innovació educativa, 10, 108121.

DE ALMEIDA, G., \& O'BRIEN, S. (2010). Analysing post-editing performance: Correlations with years of translation experience. In: Proceedings of the 14th Annual Conference of the European Association for Machine Translation.

DILLON, S., \& FRASER, J. (2006). Translators and TM: An investigation of translators' perceptions of translation memory adoption. Machine Translation, 20(2), 67-79.

DIÑO, G. (2016). Baidu Translate: The Inside Story. Retrieved from https://slator.com/technology/baidu-translate-the-inside-story/

DUCAR, C., \& SCHOCKET, D. H. (2018). Machine translation and the L2 classroom: Pedagogical solutions for making peace with Google translate. Foreign Language Annals, 51(4), 779795.

FIEDERER, R., \& O’BRIEN, S. (2009). Quality and machine translation: A realistic objective. The Journal of Specialised Translation, 11(11), 52-74.

GARCÍA, I. (2010). Is machine translation ready yet? Target, 22(1), 7-21.

GARCÍA, I. (2011). Translating by post-editing: is it the way forward? Machine Translation, 25(3), 217. 
GASPARI, F. (2001). Teaching machine translation to trainee translators: A survey of their knowledge and opinions. MT Summit VIII Workshop on Teaching Machine Translation, 35-44. http://mt-archive.info/MTS-2001-Gaspari.pdf

GOOGLE (2016). A Neural Network for Machine Translation, at Production Scale. Retrieved from https://ai.googleblog.com/2016/09/a-neural-network-for-machine.html

GRACE, K. ET AL. (2018). When will AI exceed human performance? Evidence from AI experts. arXiv:1705.08807

GUERBEROF ARENAS, A. (2009). Productivity and quality in MT post-editing, In Proceedings of the MT Summit XII workshop on "Beyond Translation Memories: New tools for translators". AMTA.

GUERBEROF ARENAS, A. (2013). What do professional translators think about post-editing? The Journal of Specialized Translation, 19, 75-95.

GUERBEROF ARENAS, A., \& MOORKENS, J. (2019). Machine translation and post-editing training as part of a master's programme. The Journal of Specialised Translation, 31, 217238.

KENNY, D., \& DOHERTY, S. (2014). Statistical machine translation in the translation curriculum: overcoming obstacles and empowering translators. The Interpreter and Translator Trainer, 8(2), 276-294.

HARTLEY, T. \& SCHUBERT, K. (1998). From testbench to workflow: Relocating MT in education and training. In Translating and the computer, 20. Proceedings from the Aslib conference held on 12 \& 13 November 1998, London. Londres: Aslib

HASSAN, H., AUE, A., CHEN, C., CHOWDHARY, V., CLARK, J., FEDERMANN, C., HUANG, X., JUNCZYS- DOWMUNT, M., LEWIS, W., LI, M., LIU, S., LIU, T.- Y., LUO, R., MENEZES, A., QIN, T., SEIDE, F., TAN, X., TIAN, F., WU, L., ...ZHOU, M. (2018). Achieving human parity on automatic Chinese to English news translation. www.microsoft.com/ en- us/ research/ uploads/ prod/ 2018/ 03/ final- achieving- human.pdf

HAUGH, M. (2017). Translation technology is useful but should not replace learning languages. The Conversation October 16. https://theconversation.com/translationtechnology-is-useful-but-should-not-replace-learning-languages-85384

JOLLEY, J. R., \& MAIMONE, L. (2015). Free online machine translation: Use and perceptions by Spanish students and instructors. In A. J. MOELLER (ed.) Learn languages, explore cultures, transform lives (pp. 181-200). Egg Harbor, WI: Central States Conference on the Teaching of Foreign Languages.

KATAN, D. (2011). Occupation or profession: A survey of the translators' world. In R. SELASHEFFY \& M. SHLESINGER (Eds.), Identity and status in the translational professions (pp. 65-87). Amsterdam: John Benjamins.

KENNY, D., \& WINTERS, M. (2020). Machine translation, ethics and the literary translator's voice. Translation Spaces, 9(1), 123-149.

KIRALY, D. (2000). A social constructivist approach to translator education. Empowerment from theory to practice. Manchester: St Jerome.

KOPONEN, M. (2016). Is machine translation post-editing worth the effort? A survey of research into post-editing and effort. The Journal of Specialised Translation, 25, 131-148. 
KOSKINEN, K., \& RUOKONEN, M. (2017). Love letters or hate mail? Translators' technology acceptance in the light of their emotional narratives. In D. KENNY (Ed.), Human issues in translation technology (pp. 26-42). Nueva York: Routledge.

LÄUBLI, S., SENNRICH, R. \& VOLK, M. (2018). Has machine translation achieved human parity? A case for document-level evaluation. https://arxiv.org/pdf/1808.07048.pdf

LEBLANC, M. (2013). Translators on Translation Memory (TM). Results of an ethnographic study in three translation services and agencies. Translation \& Interpreting, 5(2), 1-13.

LEBLANC, M. (2014). Les mémoires de traduction et le rapport au texte : ce qu'en disent les traducteurs professionnels. TTR, 27(2), 123-148.

LEWIS, D. (1997) Machine translation in a Modern Languages curriculum. Computer Assisted Language Learning, 10(3), 255-271.

MOORKENS, J. (2018). What to expect from Neural Machine Translation: a practical in-class translation evaluation exercise. The Interpreter and Translator Trainer, 12(4), 375-387.

NIÑO, A. (2008) Evaluating the use of machine translation post-editing in the foreign language class. Computer Assisted Language Learning, 21(1), 29-49.

NIÑO, A. (2009). Machine translation in foreign language learning: Language learners' and tutors' perceptions of its advantages and disadvantages. ReCALL, 21(2), 105-122.

O'BRIEN, S. (2002). Teaching post-editing: A proposal for course content. 6th EAMT Workshop Teaching machine translation, 14-15 November 2002, 99-106. http://www.mt-archive.info/00/EAMT-2002-OBrien.pdf

O'BRIEN, S. (2007). An empirical investigation of temporal and technical post-editing effort. Translation and Interpreting Studies, 2(1), 83-136.

PLITT, M., \& MASSELOT, F. (2010). A productivity test of statistical machine translation postediting in a typical localisation context. Prague Bulletin of Mathematical Linguistics, 93, 7-16.

PYM, A. \& TORRES-SIMÓN, E. (2016). Designing a course in Translation Studies to respond to students' questions. The Interpreter and Translator Trainer, 10 (2), 183-203.

PYM, A. \& TORRES-SIMÓN, E. (2021). Efectos de la automatización en las competencias básicas del traductor: la traducción automática neuronal. In A. VIDAL SUÑÉ \& A. ALARCÓN ALARCÓN (Eds.) Ocupaciones y lenguaje. Indicadores y análisis de competencias lingüísticas en el ámbito laboral (pp. 479-509). Tarragona: Publicacions URV.

ROTHWELL, A., \& SVOBODA, T. (2018). Tracking translator training in tools and technologies: Findings of the EMT survey 2017. Journal of Specialised Translation, 32, 26-60.

TATSUMI, M. (2009). Correlation between automatic evaluation metric scores, post-editing speed, and some other factors. In Proceedings of the MT-Summit XII (pp. 332-339). AMTA.

TEIXEIRA, C. D. S. (2014) The impact of metadata on translator performance: how translators work with translation memories and machine translation. Tesis doctoral. Universitat Rovira i Virgili.

TEMIZÖZ, Ö. (2013) Postediting machine-translation output and its revision. Subject-matter experts versus professional translators. Tesis doctoral. Universitat Rovira i Virgili.

VIEIRA, L. N. (2018). Human challenges in the use of machine translation in professional translation processes. In ITI Research Network e-book 2018: The Human and the Machine (p.7). Institute of Translation and Interpreting. 
WOOD, E. (2018). Twenty Years of Building for Everyone. Company Announcements. https://www.blog.google/inside-google/company-announcements/twenty-yearsbuilding-everyone/. 